\title{
Impaired Pulmonary Perfusion Associated With Thymus Hyperplasia in an Infant Candidate for Fontan Operation
}

\author{
Hirotaka Ishido, MD; Satoshi Masutani, MD; Hideaki Senzaki, MD
}

\begin{abstract}
An enlarged thymus adversely affected pulmonary artery development and perfusion in a 4-month-old boy with asplenia syndrome and a single ventricular circulation with aorto-pulmonary shunt. Steroid administration for 4 weeks successfully reduced thymus volume, concomitant with improvement of pulmonary vasculature and perfusion. Given the importance of pulmonary physiology in the Fontan circulation, the effects of thymic hyperplasia on pulmonary circulation should be taken into consideration in the overall management of candidates for Fontan surgery. (Circ J 2009; 73: 2348-2351)
\end{abstract}

Key Words: Congenital heart disease; Fontan procedure; Risk factor; Surgery

$\mathbf{T}$ hymus hyperplasia is not a rare condition in infancy, and is generally considered to be asymptomatic. On rare occasions, however, it can cause respiratory distress by displacing and/or compressing the bronchopulmonary system. ${ }^{1,2}$ We report that enlarged thymus adversely affected pulmonary artery growth and perfusion in a 4-month-old boy who was a candidate for Fontan surgery. Because the development of the pulmonary vascular bed to an adequate size and function is one of the most important factors in accomplishing successful Fontan procedure in patients with single ventricular circulation, ${ }^{3-5}$ this case report is an important message to physicians involved in the management of patients with a single ventricle.

\section{Case Report}

The patient had a single right ventricle and pulmonary atresia complicated with asplenia syndrome, and underwent aorto-pulmonary shunt operation at the age of 3 weeks. He subsequently showed a gradual decrease in oxygen saturation without respiratory distress during follow-up at the outpatient clinic. Chest X-ray taken at the age of 4 months showed an apparent decrease in air volume in the right apical area of the lung (Figure 1A). Computed tomography showed an enlarged thymus that occupied the right upper space, corresponding to the X-ray finding (Figure 2). Considering the potential adverse effects of a hyperplasic thymus on the pulmonary circulation, the patient underwent cardiac catheterization to evaluate the pulmonary vascular bed. There was a considerable decrease in the right upper peripheral pulmonary arteries compared with those of the left side, as demonstrated on pulmonary angiography (Figure 3A). Pulmonary perfusion scintigraphy confirmed a significant decrease in right pulmonary perfusion with a right-to-left perfusion ratio of 31:100, consistent with the angiographic findings (Figure 4A).

Because the thymus tissue on CT was homogeneous and
A

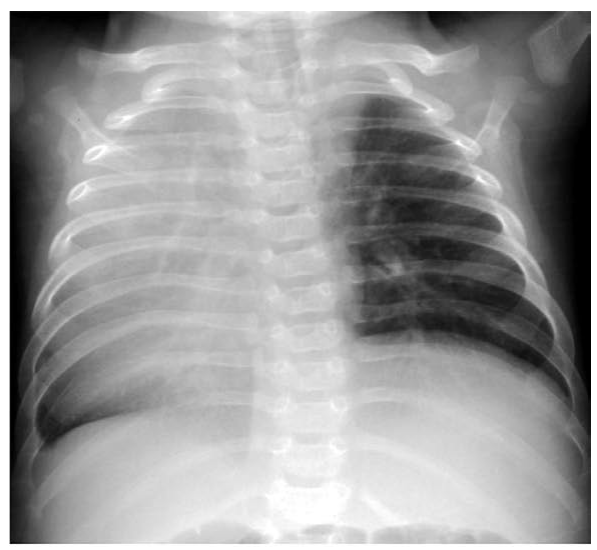

B

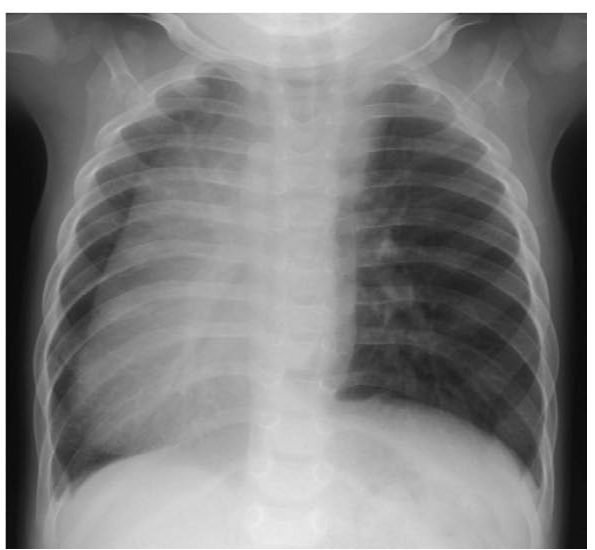

Figure 1. (A) Chest X-ray showing decreased air volume in the right apical area of the lung. (B) Chest X-ray showing the reduction in thymus volume with improved air volume in the right apical area of the lung after steroid therapy.

Received October 7, 2008; accepted February 17, 2009; released online June 3, 2009

Department of Pediatric Cardiology and Pediatrics, International Medical Center, Saitama Medical University, Saitama, Japan

Mailing address: Hideaki Senzaki, MD, Department of Pediatric Cardiology, Saitama Heart Institute, Saitama Medical University Hospital,

38 Morohongo, Moroyama, Saitama 350-0495, Japan. E-mail: hsenzaki@ saitama-med.ac.jp

All rights are reserved to the Japanese Circulation Society. For permissions, please e-mail: cj@j-circ.or.jp 

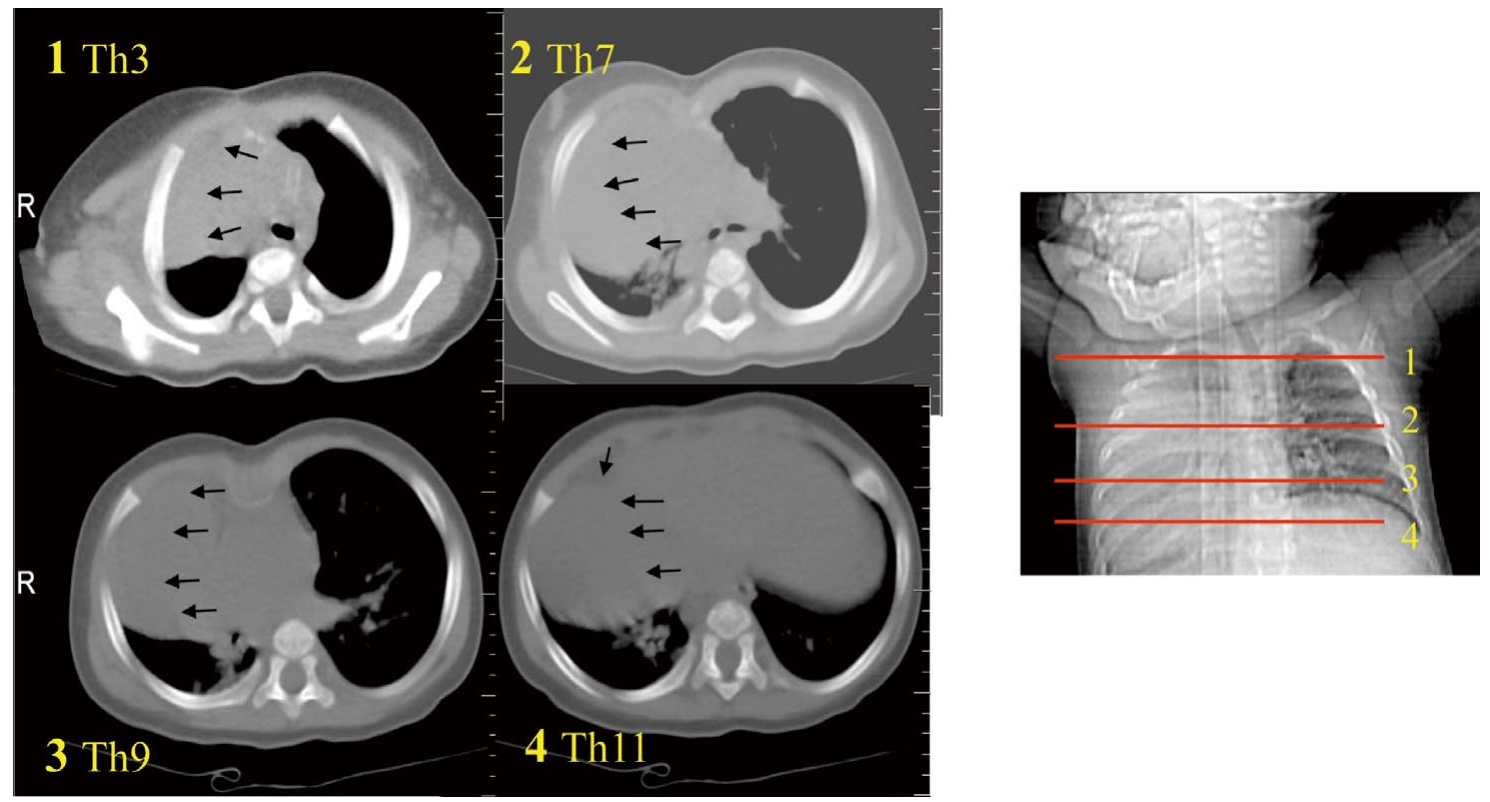

Figure 2. Computed tomography findings before steroid therapy showing occupation of the right apical space by the enlarged thymus. Slice levels are indicated on the right-hand side. In each slice, the thymus tissue boundary is indicated by arrows. Th, thoracic spine.

A

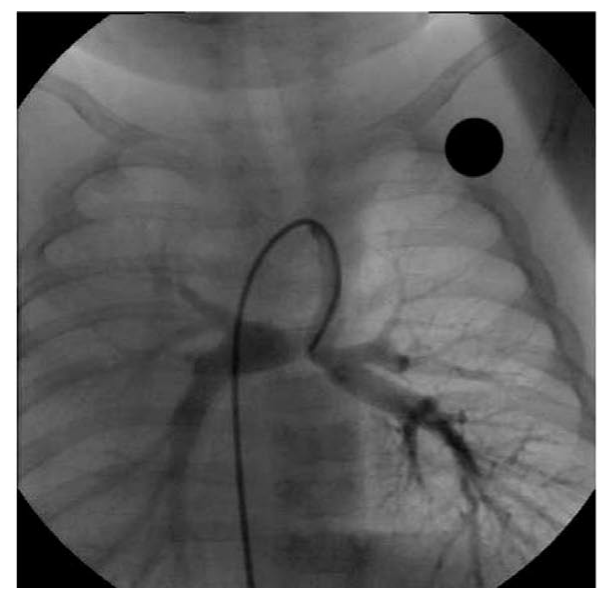

B

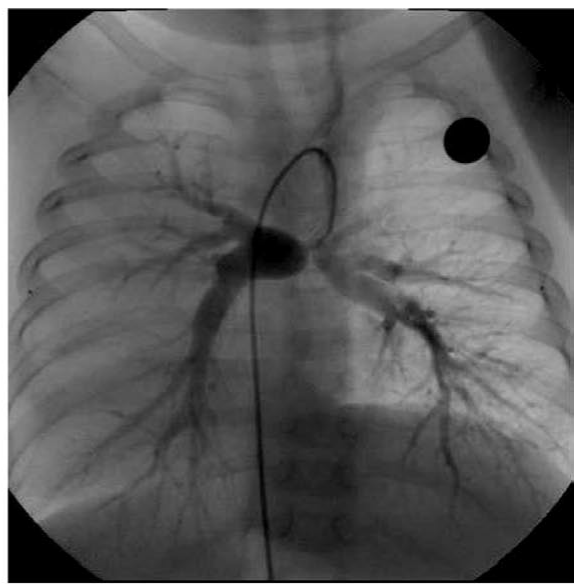

Figure 3. Pulmonary angiography before $(\mathbf{A})$ and after (B) reduction of thymus volume by steroid therapy. Although the thymus itself may affect the contrast of lung air and the pulmonary artery, and the projections, figure positions and diaphragm levels in each flame are different between the 2 angiograms, reduction of thymus volume appeared to improve the right upper pulmonary vasculature.

because there were no findings indicative of invasion of other tissues, we assumed that the enlarged thymus represented physiologic hyperplasia of normal thymic tissue. We therefore started oral treatment with steroid $\left(1 \mathrm{mg} \cdot \mathrm{kg}^{-1} \cdot\right.$ day $^{-1}$ of predonine for 2 weeks followed by $0.5 \mathrm{mg} \cdot \mathrm{kg}^{-1} \cdot \mathrm{day}^{-1}$ for another 2 weeks) with the aim of reducing thymus tissue volume; 4 weeks later, chest X-ray showed a dramatic reduction in thymus volume (Figure 1B), concomitant with improvement in arterial oxygen saturation (from $72 \%$ to $82 \%$ at cardiac catheterization before and after steroid therapy) and right pulmonary perfusion as indicated by pulmonary perfusion scintigraphy (Figure 4B; right-to-left perfusion ratio, 59:100). Pulmonary angiography also showed an improvement in the right upper pulmonary vasculature (Figure 2). There were no complications associ- ated with steroid therapy, and the patient successfully underwent bi-directional Glenn anastomosis at the age of 7 months and subsequent Fontan operation at the age of 2 years. At the time of surgery, the thymus tissue appeared normal, and there has been no recurrence to date (4 years of age) of thymus enlargement, providing support to our provisional diagnosis that the enlargement was physiologic hyperplasia of the thymus gland.

\section{Discussion}

The most common cause of thymic enlargement in children is physiologic hyperplasia, but it is usually a coincidental finding rather than the cause of pathologic signs and symptoms. Symptomatic thymic enlargement, although rare, has 
A
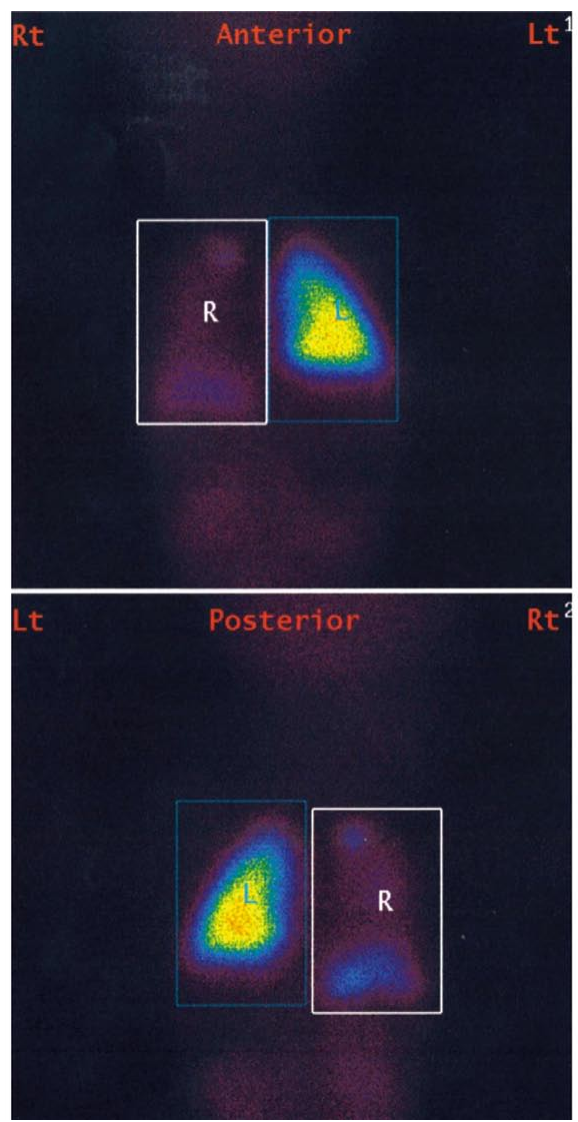

B
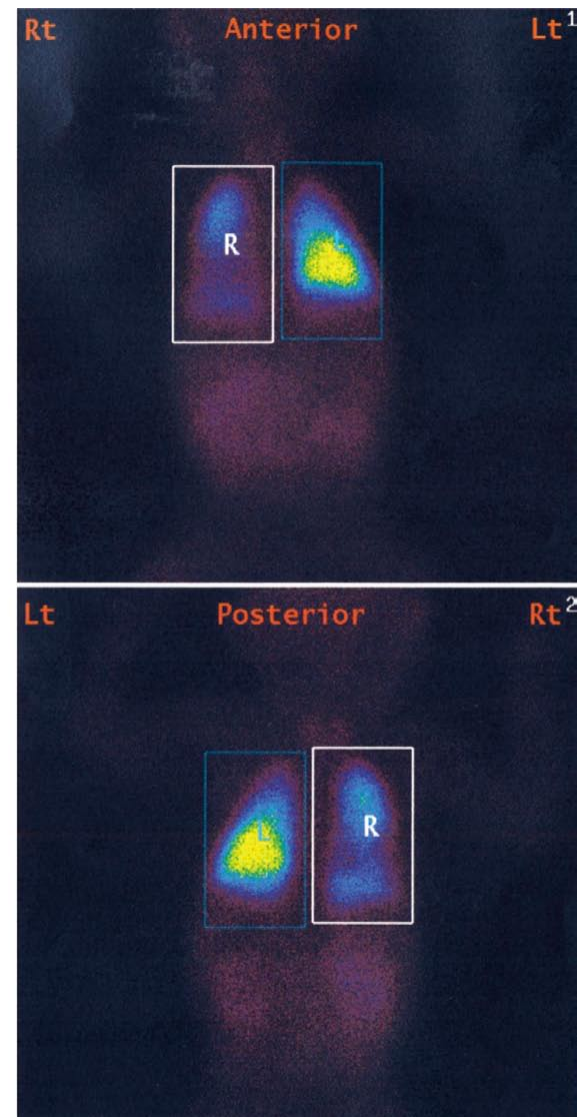

Figure 4. Pulmonary perfusion scintigraphy demonstrating a significant decrease in right pulmonary perfusion (A), which was improved after thymus reduction with steroid therapy (B). Both anterior (Upper panels) and posterior (Lower panels) projections are demonstrated. Anterior, posterior and average right (R) -to-left (L) perfusion ratios were improved from 25.9:100 to 53.7:100, from 37.2:100 to 64.2:100, and from 31.2:100 to 58.9:100, respectively.

been reported as always associated with respiratory symptoms. $1,2,6$ To the best of our knowledge, the present case is the first to demonstrate that a hyperplastic thymus has the potential to cause impaired pulmonary artery growth and perfusion during childhood growth. The significance of the adverse effect of thymus hyperplasia on the pulmonary circulation in the present patient was supported by the improvement in arterial saturation, angiographic findings and pulmonary perfusion following the reduction of thymus volume by steroid therapy. The patient's background (the source of pulmonary blood flow was aorto-pulmonary shunt rather than an ejecting ventricle and he had an asplenia syndrome) may have had a synergistic influence on the pulmonary circulation. If the patient had had a double ventricular system in which pulsatile pulmonary blood flow was supplied by right ventricular contractile energy, right pulmonary perfusion might have been maintained despite the enlarged thymus. In addition, dextrocardia associated with asplenia syndrome might further amplify the effect of thymus hyperplasia on the right pulmonary circulation in this patient. Because low pulmonary artery resistance is a prerequisite for establishing successful Fontan circulation where there is no ventricle to propel blood flow into the pulmonary circulation, ${ }^{3}$ any condition that could impede pulmonary blood flow, such as pulmonary hypoplasia, stenosis or distortion, must be corrected before the Fontan procedure. $^{7}$ The present report indicates that thymus hyperplasia could cause pulmonary flow impediment in Fontan can- didates and thus could be a therapeutic target in the overall management of patients scheduled for Fontan surgery. Also, it should be noted that there was still laterality of perfusion even after the reduction of thymus volume with steroid in this patient. Because adequate blood flow is required for the growth of the vascular bed, a further period of time would be needed for normalization of the pulmonary vasculature and resultant improvement of pulmonary flow distribution, highlighting the importance of early intervention for an enlarged thymus.

In this patient, steroid administration for 4 weeks successfully reduced the thymus volume. Previous reports, however, indicated that steroid therapy hads inconsistent results in shrinking a hyperplastic thymus gland, ${ }^{8,9}$ often requiring surgical excision. Nonetheless, we first selected steroid therapy, because the patient's condition was not urgent and because we hoped to avoid median sternotomy, which will be needed in the subsequent Glenn and Fontan surgeries. Thus, for Fontan candidates, we believe that steroid therapy is worth trying as the first choice for reducing thymus volume to improve pulmonary artery development and perfusion.

In summary, a hyperplastic thymus can impair pulmonary artery growth and perfusion in patients with a single ventricle. Given the importance of the pulmonary physiology in the Fontan circulation, the effects of thymic hyperplasia on the pulmonary circulation should be taken into consideration in the overall management of candidates for Fontan surgery. 


\section{Acknowledgments}

Supported by Grants from Nipro Corporation (H.S.), Kawano Memorial Foundation: No. 10-3 (H.S.), and Tensindo Medical Institution (H.S.).

\section{References}

1. Linegar AG, Odell JA, Fennell WM, Close PM, De Groot MK, Casserly DR, et al. Massive thymic hyperplasia. Ann Thorac Surg 1993; 55: 1197-1201.

2. Pedroza Melendez A, Larenas-Linnemann D. Thymus hyperplasia: Differential diagnosis in the wheezing infant. Allergol Immunopathol 1997; 25: 59-62.

3. Senzaki H, Isoda T, Ishizawa A, Hishi T. Reconsideration of criteria for the Fontan operation: Influence of pulmonary artery size on postoperative hemodynamics of the Fontan operation. Circulation 1994; 89: $1196-1202$
4. Kawazu Y, Inamura N, Kayatani F. Prediction of therapeutic strategy and outcome for antenatally diagnosed pulmonary atresia/stenosis with intact ventricular septum. Circ J 2008; 72: 1471-1475.

5. Senzaki H, Naito C, Kobayashi T, Sasaki N, Kyo S, Yokote Y, et al. Influence of age (body size) on the Fontan circulation: Analysis by a theoretical model. Jpn Circ J 2000; 64: 943-948.

6. Rubb ME, Keilani R, Howatson AG, Haddad N, Remani H. Benign symptomatic thymic tumors. J Pediatr Surg 2000; 35: 1362-1364.

7. Fontan F, Fernandez G, Costa F, Naftel DC, Tritto F, Blackstone EH, et al. The size of the pulmonary arteries and the results of the Fontan operation. J Thorac Cardiovasc Surg 1989; 98: 711-719; discussion 719-724.

8. Lamesch AJ. Massive thymic hyperplasia in infants. $Z$ Kinderchir 1983; 38: 16-18.

9. Kobayashi T, Hirabayashi Y, Kobayashi Y. Diagnostic value of plain chest roentgenogram and CT scan findings in four cases of massive thymic hyperplasia. Pediatr Radiol 1986; 16: 452-455. 